\title{
The Value and Application of Personalized Needle Modification in Percutaneous Infrazygomatic Radiofrequency in Isolated Maxillary Nerve Pain through the Foramen Rotundum
}

Yajing Chen, MD',2, Qiang Zhu, MD³, Bing Huang, MD², Qianying Liu, MD', Qiuli He, MD1, Yibing Yao, BS², Kang An, MD${ }^{4}$, Chunyan Zhu, MD², Wei Chen, $\mathrm{MD}^{2}$, and Ming Yao, $\mathrm{PhD}^{2}$

From: ${ }^{1 B e n g b u}$ Medical College, Bengbu, Anhui Province, China; ${ }^{2}$ First Affiliated Hospital of Jiaxing University, Jiaxing, Zhejiang Province, China; ${ }^{3}$ Tiantan Hospital, Beijing, China; ${ }^{4}$ Gulou Hospital, Nanjing, Jiangsu Province, China

Address Correspondence: Bing Huang, MD Department of

Anesthesiology and Pain Medical Center First Affiliated Hospital of liaxing University 1882 Zhonghuan South Road

Jiaxing 314001, China E-mail: 601538729@qq.com

Disclaimer: There was no external funding in the preparation of this manuscript.

Conflict of interest: Each author certifies that he or she, or a member of his or her immediate family, has no commercial association

(i.e., consultancies, stock ownership, equity interest, patent/licensing arrangements, etc.) that might pose a conflict of interest in connection with the submitted manuscript.

Manuscript received: 11-03-2018

Revised manuscript received: 12-21-2018

Accepted for publication: 02-07-2019

Free full manuscript: www.pain physicianjournal.
Background: Percutaneous infrazygomatic radiofrequency (PIR) is a common approach used to block isolated maxillary nerve (V2) pain through the foramen rotundum (FR) in patients with trigeminal neuralgia (TN). Nevertheless, when using this method, there is a risk of accidental penetration of the superior orbital fissure (SOF) and the optic canal (OC) that may result in the injury of the vessels and nerves in that area, and in some severe cases may lead to blindness. According to the blocking of the external orifice of the FR and whether a curved needle was used, combined angle deviation from the path of percutaneous infrazygomatic approach, the FR to the SOF or the OC in the treatment of V2 pain, in which no research has reported the angle, we analyzed the value and application of personalized needle modification in PIR in isolated maxillary nerve pain through the FR.

Objectives: The following study examined the relationship between the FR and the SOF, and analyzed the clinical significance of personalized needle modification in computed tomography (CT)-guided PIR ablation of the maxillary nerve through the FR in patients with TN.

Study Design: Randomized, review, clinical research study.

Setting: Department of Anesthesiology and Pain Medical Center, Jiaxing, China.

Methods: Three-dimensional reconstruction was performed in 88 patients and 136 patients with PIR ablation in isolated maxillary nerve pain through the FR. According to the blocking of the external orifice of the FR and whether a curved needle was used, patients were divided into 4 groups: curvedneedle blocking group (CB), straight-needle blocking group (SB), straight needle no-blocking group $(\mathrm{SN})$, and curved-needle no-blocking group (CN).

Results: The results obtained revealed minimum $\mathrm{H}$ (shortest diameter of the $\mathrm{FR}$ ) $=1.0 \mathrm{~mm}$ and minimum $L$ (length of the FR tubes) $=3.7 \mathrm{~mm}$. The distance between the external orifice of the $F R$ and the SOF (FS) was $5.16 \pm 1.33 \mathrm{~mm}$. The angle A (between the radiofrequency needle and the sagittal plane) was $39 \pm 3.95^{\circ}$; the angle between the canthomeatal line and the CT scan line (ACT) was 58.99 $\pm 6.23^{\circ}$; the puncture depth (LS) was $63.99 \pm 4.24 \mathrm{~mm}$; the deviation angle of the misplacement into the SOF (SAF) was $2.96 \pm 0.71^{\circ}$; the deviation angle of the misplacement into the OC (OAF) was 4.95 $\pm 0.73^{\circ}$. In addition, the postoperative Numeric Rating Scale scores in the CB group were significantly lower compared with the SB group, whereas the probability of entering the SOF in the CB group was significantly lower compared with the SB group. The total number of punctures in the SN group was less than that in the CN group.

Limitations: Additional clinical data should be collected to preserve the results in future work.

Conclusions: The distance between the FR and the SOF or the OC was only few millimeters, and slight angle error could lead to the SOF and the OC. For patients with blockage in the path, the treatment of radiofrequency with personalized needle modification could improve the curative effect and reduce the risk of accidental SOF penetration.

Key words: Trigeminal neuralgia, foramen rotundum, superior orbital fissure, radiofrequency, personalized needle modification

Pain Physician 2019: 22:377-387 
T rigeminal neuralgia (TN), a chronic neuropathic pain with unclear pathogenesis, is often described by recurrent electric shocks or sharp shooting pain caused by nonpainful facial movement or stimuli, such as speaking, brushing teeth, face washing eating, and similar tasks (1-5). TN always affects one or more branches of the trigeminal nerve, with V2 at $17 \%, \mathrm{~V} 3$ at $15 \%, \mathrm{~V} 1$ at $4 \%$, and V2 + V3 approximately $32 \%$ (6) of the time. The attacks may be experienced for days, weeks, or months and in some cases may occur many times per day, seriously affecting the patient's daily life. Oral antiepileptic drugs are the conservative treatment for TN. If this treatment approach is ineffective or intolerable, alternative invasive methods are used such as craniotomy microvascular decompression (7), percutaneous radiofrequency therapy of the Gasserian ganglion $(8,9)$, balloon compression (10), injection of drugs that can induce chemical damage (11), and gamma knife $(12,13)$. Radiofrequency treatment is a minimally invasive, low-risk method for treating TN with a high rate of initial efficacy and lower frequent side effects $(8,9,14,15)$. Every approach has its own risk. Percutaneous trans-foramen ovale (FO) approach to the Gasserian ganglion is a typical method; however, approach to the $\mathrm{FO}$ is a difficult and time-consuming procedure that requires precision to place the needle near the Gasserian ganglion, and could lead to intracranial hemorrhage, subarachnoid injection, infection, and others (16). Percutaneous infrazygomatic radiofrequency (PIR) is a common method used to block the isolated maxillary nerve (V2) through the foramen rotundum (FR) in patients with TN; PIR offers a higher rate of pain relief and higher safety and branch selectivity (15). Nevertheless, there is a risk of accidental entry into the superior orbital fissure (SOF) and the optic canal $(O C)$, which may result in the injury of the vessels and nerves in the SOF and the OC, and in some extreme cases may result in blindness. Currently, there are no reports on the angle deviation from the path of PIR to the FR with the SOF or the OC. The aim of the present study was to examine the possibility of angle deviation from the path of percutaneous infrazygomatic approach to the FR to the SOF or the $O C$ in the treatment of V2 pain. We found that the puncture needle was blocked by the large wing of the sphenoid bone at the external orifice of the FR when some patients were punctured into the FR (17), which not only increased the puncture difficulty, but also forced the needle tip up into the SOF when it crossed the barrier. We collected clinical data on the blocking of the external orifice of the FR in relation to the puncture path and in relation to whether the curved needle technique was used or not. In addition, we examined whether there was any influence on the probability of entering into the SOF, to guide the clinical work and reduce the incidence of nerve and vascular injury in the SOF and the OC.

This is a randomized, review, clinical research study. The following study examined the relationship between the FR and the SOF, and analyzed the clinical significance of personalized needle modification in computed tomography (CT)-guided PIR ablation of the maxillary nerve through the FR in patients with TN.

\section{Methods}

\section{CT Scan and Three-Dimensional Reconstruction}

A total of 88 (176 sides) CT (Siemens Somatom Sensation 64 spiral scanner) scans of nose and paranasal sinuses from adult patients and 3-dimensional (3D) reconstructions were collected from our hospital between January 2018 and June 2018. There were 28 male patients and 60 female patients aged 19-88 years with an average age of 48.73 years. The inclusion criteria were the following: 1) 64-row high-resolution CT (HRCT) scan and 3D reconstruction of the paranasal sinus; 2) the FR, the SOF, and the OC were clearly displayed and recognized; and 3) no previous history of traumatic fracture of skull base. Exclusion standards were: 1) structural deformation caused by malignant tumor invading the FR and its surrounding structure; 2 ) previous history of traumatic fracture of skull base; 3 ) the FR that was unclear or difficult to discern; and 4) CT scan that did not reach the planned point of puncture at the lower edge of the zygomatic arch. A flow chart of study patients and relevant data measurements and analyses are shown in Table 1.

Siemens Somatom Sensation 64 HRCT scanning was performed as follows: patient was in supine position; scanning range was supraorbital glabellar to superior alveolar. Scanning parameters were slice: 0.6 $\mathrm{mm}$; kernel H41: medium +; window: sinuses 2; field of view: $178 \mathrm{~mm}$; center X: $0 \mathrm{~mm}$; center Y: $-50 \mathrm{~mm}$; mirroring: none; begin position: $127.5 \mathrm{~mm}$; end position: $227.5 \mathrm{~mm}$; image order: caudocranial; increment: 0.6 $\mathrm{mm}$; number of images: 167 . Next, the bone window was acquired, the 3D reconstruction was constructed, and finally, the image was analyzed. Measurements of 88 cases were analyzed using the software measuring 


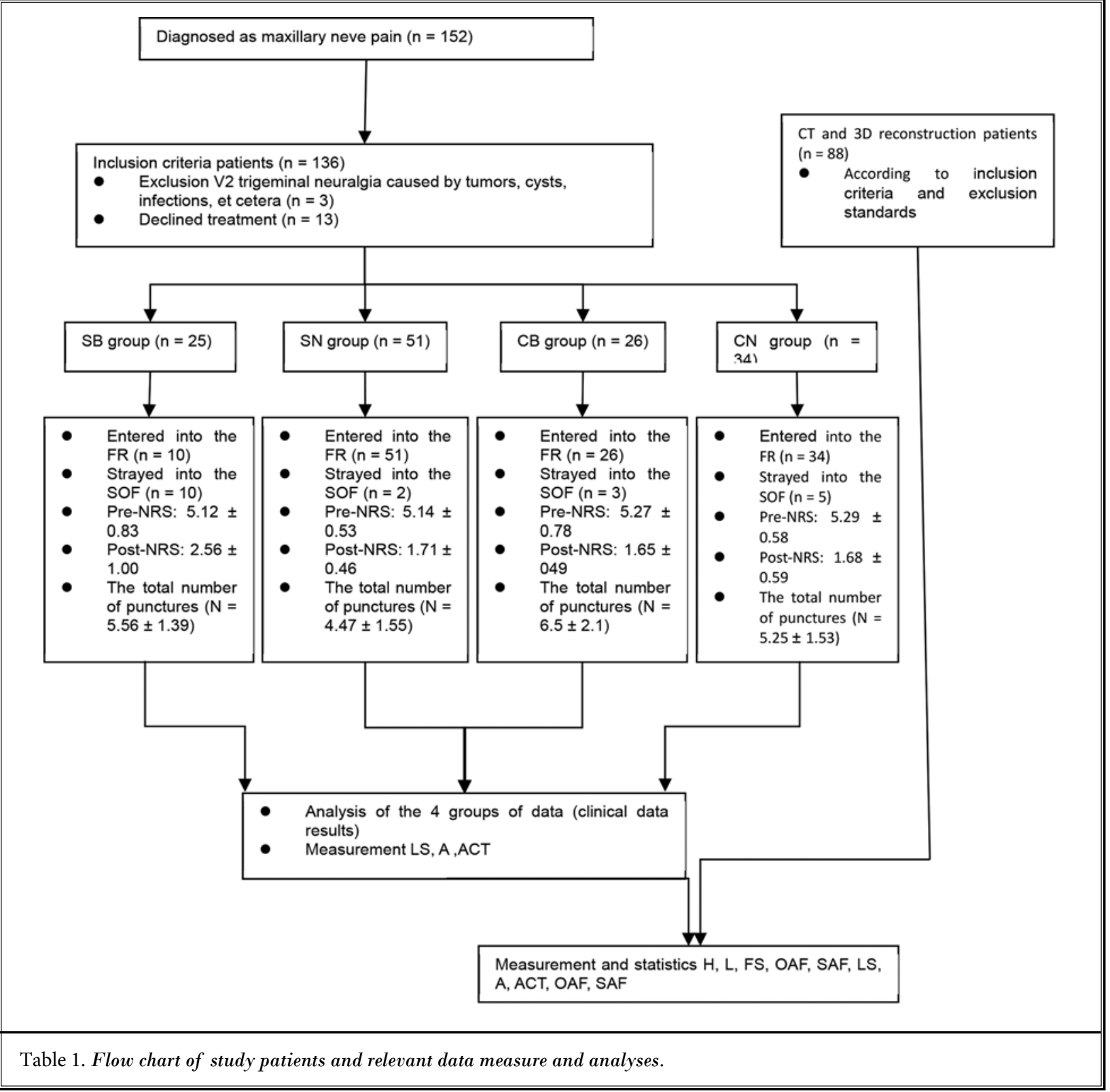

tool. The 2-dimensional measurement is shown in Fig. 1 , whereas the 3D reconstruction is shown in Fig. 2.

\section{Clinical Research Object}

The study included patients with maxillary nerve pain who were diagnosed with primary TN according to the International Association for the Study of Pain criteria in our hospital from March 2015 to June 2018, and were expected to be treated under the guidance of $\mathrm{CT}$ half coronal scanning through percutaneous infrazy- gomatic to FR radiofrequency ablation. Inclusion criteria were the following: 1) patients diagnosed with maxillary nerve pain; 2) those without mental illness who were able to cooperate with surgery and complete the Numeric Rating Scale (NRS) score (assessing pain levels over the last 24 hours; 0: painless; 1-3: mild pain, does not affect sleep; 4-6: moderate pain: 7-10: severe pain, unable to fall asleep or painful waking during sleep); 3) those who do not respond to oral antiepileptic drugs or cannot tolerate it; 4 ) duration $\geq 6$ months, NRS score 


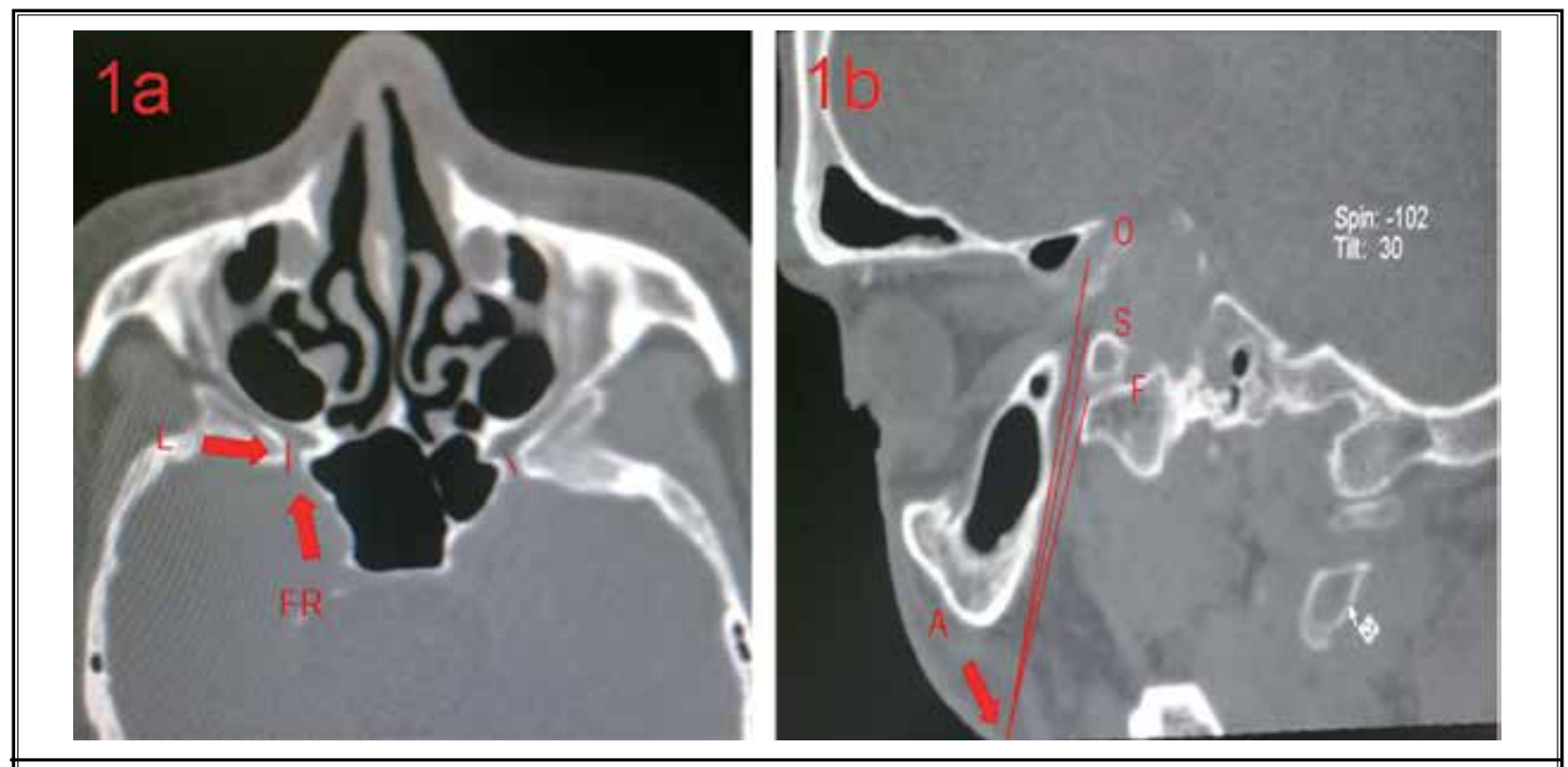

Fig. 1. Two-dimensional measurement. (A) Transverse position; L: the length of the FR. (B) Half sagittal position; A: preparation of puncture point; $O A F$ : angle of deviation from the FR to the $O C$; $S A F$ : angle of deviation from the FR to the $S O F$.
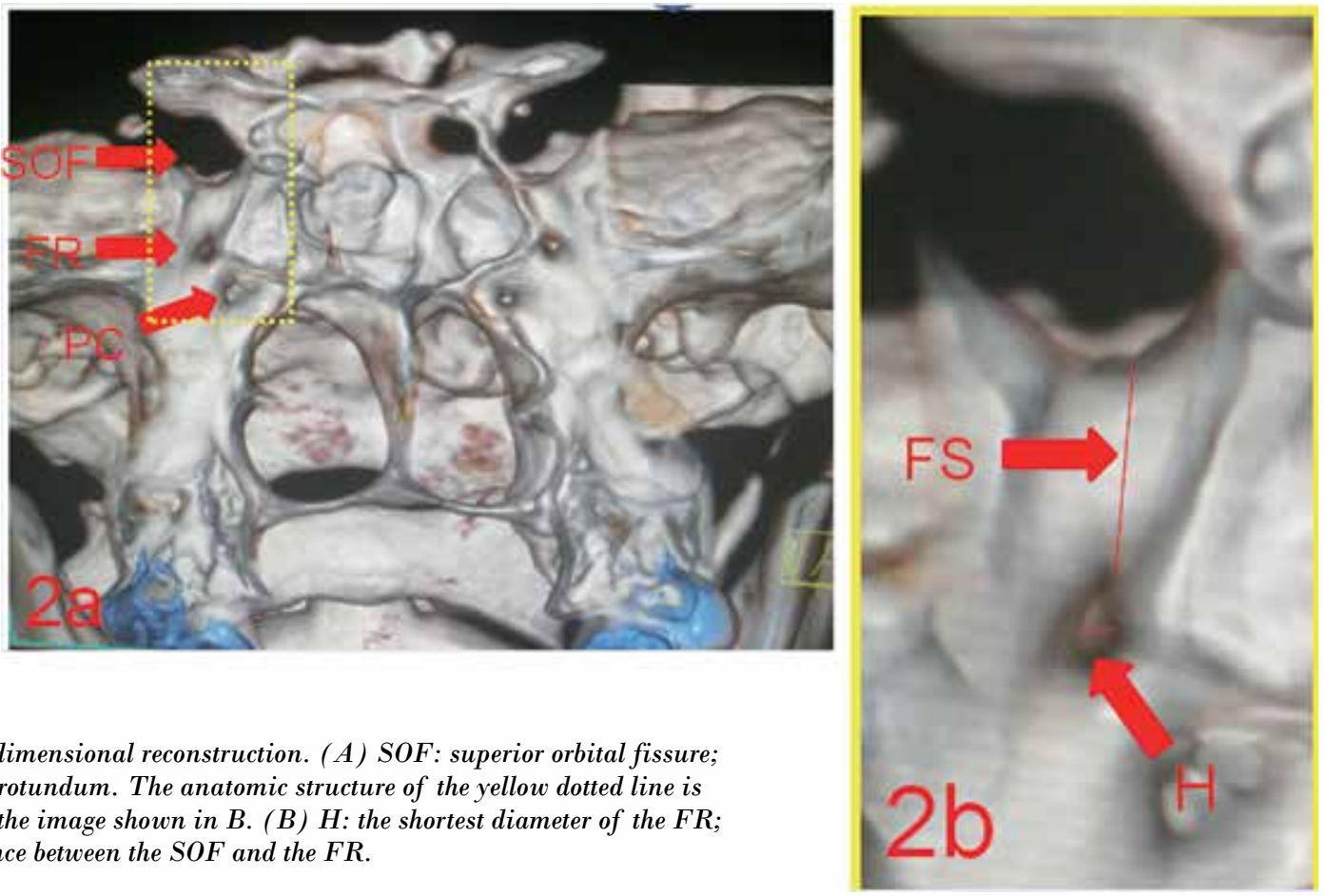

Fig. 2. Three-dimensional reconstruction. (A) SOF: superior orbital fissure; FR: foramen rotundum. The anatomic structure of the yellow dotted line is magnified by the image shown in $B$. (B) $H$ : the shortest diameter of the FR; FS: the distance between the SOF and the FR.

cian. Exclusion criteria were: 1) V2 TN caused by tumors, cysts, infections, and similar; 2) severe systemic disease; 3) use of narcotic drugs and alcohol dependence or allergies; 4) skull base malformation in which it was not $\geq 5$; 5) patients with no severe systemic disease and those able to tolerate radiofrequency thermocoagulation surgery; and 6) radiofrequency thermocoagulation surgery was performed by the same experienced physi- 
possible to recognize the FR, the SOF, and the OC; and 5) patients with mental illness who were not capable of cooperating with surgery or completing the NRS score.

\section{Clinical Case Grouping and Data}

According to inclusion and exclusion criteria, 136 patients were enrolled in this study. The male/female ratio was 40/96, aged 36-95 years, with an average age of $66.6 \pm 12.7$ years. According to the blocking of the external orifice of the FR and whether the curved needle was used or not, patients were divided into 4 groups: curved-needle blocking group (CB), straight-needle blocking group (SB), straight needle no-blocking group (SN), curved-needle no-blocking group (CN). The following statistical analyses and comparison of the data were performed for all 4 groups: preoperative NRS score (pre-NRS), postoperative NRS score (post-NRS), angle between the canthomeatal line and the CT scan line (ACT) (Fig. 3A), radiofrequency needle puncture depth (LS) (Fig. 3B), if the external orifice of the FR was blocked, and the angle between the tube axis of the FR and the puncture path was measured (Fig. 3B). Also, the radiofrequency of bent needle to the angle, which was shown in Fig. 3B, was measured with the

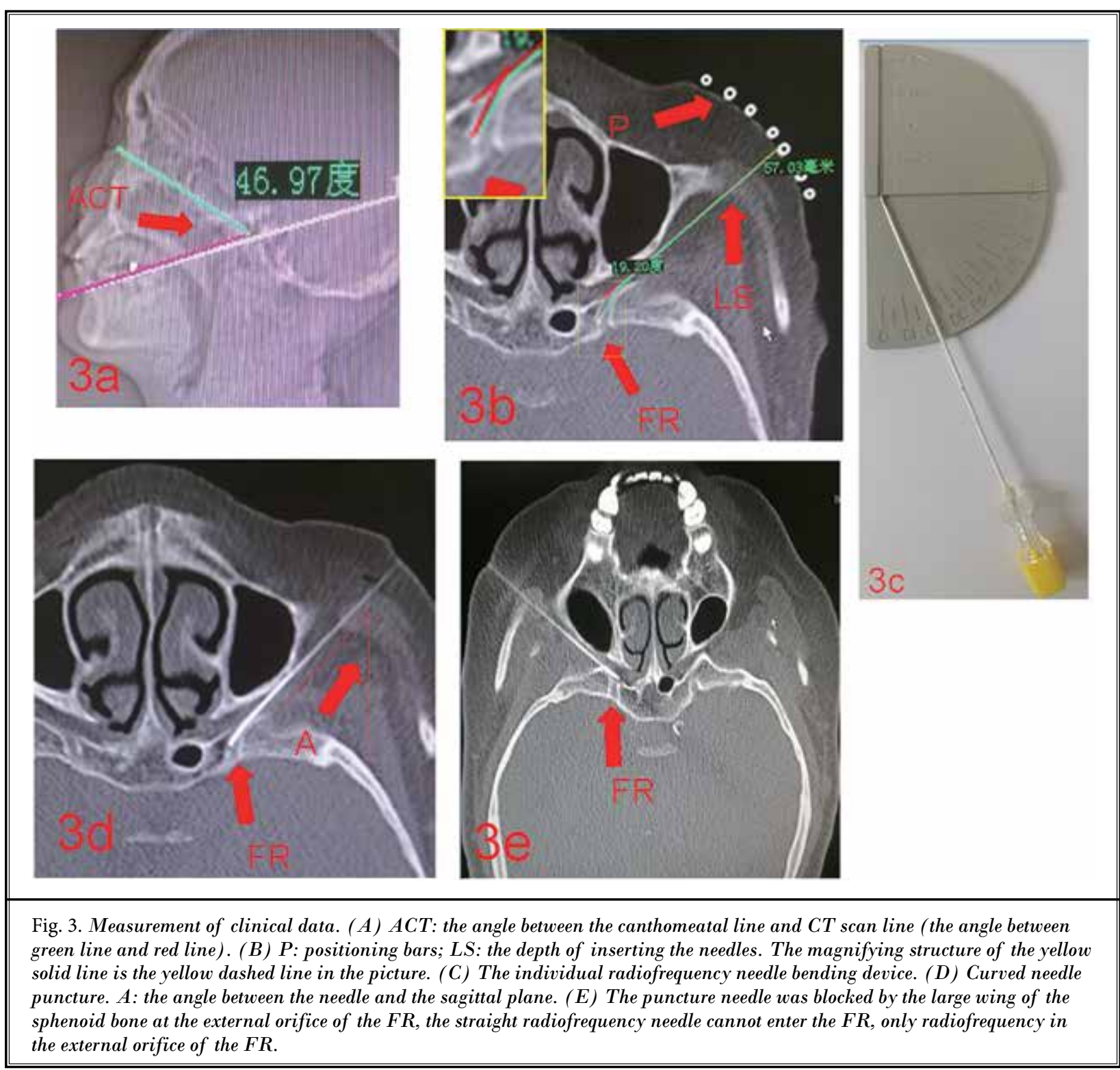




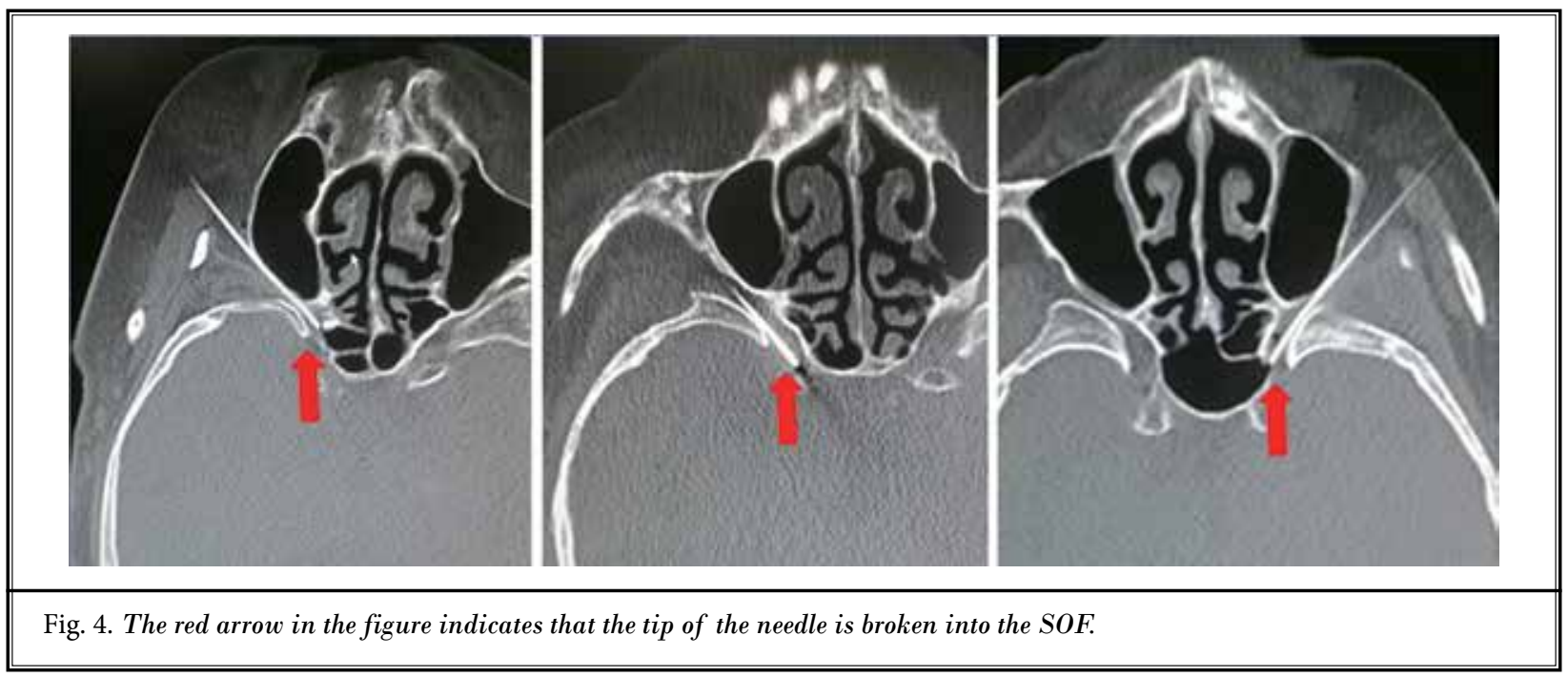

individual radiofrequency needle bending device (Fig. 3C). Then, angle $A$ between the radiofrequency needle and sagittal plane was measured (Fig. 3D), and finally, puncture with a curved personalized needle modification and radiofrequency (Fig. 3D), the total number of puncture $\mathrm{N}$ (the total adjustment time that reflects the time-consuming procedure), the ratio of not entering into the FR (Fig. $3 \mathrm{E}$ ), and the probability of the puncture needle entering into the SOF (Fig. 4).

\section{Preoperative Preparation}

Before operation, patients underwent the following examination: coagulation function analysis, liver and kidney function analysis, electrolytes testing, brain magnetic resonance imaging, paranasal sinus $\mathrm{CT}$, and electrocardiogram. Elimination of secondary TN and contraindications were also analyzed. In addition, patients fasted for 8 hours and did not consume any liquid 4 hours before the operation. Additionally, all patients were informed about potential risks and complications. We prepared related operating instruments, such as the radiofrequency therapy apparatus (Baylis Inc., Beijing, China), No. 7 radiofrequency needle (Zhejiang Volt Medical Device Co., Ltd., Zhejiang, China), positioning bars (unlabeled products), the individual radiofrequency needle bending device (Zhejiang Volt Medical Device Co., Ltd.), and obtained intravenous access, among others.

\section{Operation Steps}

With a thin pillow on the shoulder of the patient, the head was tilted back approximately $30^{\circ}$ (Fig. 3A), rotating the $\mathrm{CT}$ machine for half-coronal scanning, selecting the appropriate slices and position by the positioning bars, and marking puncture point (Fig. 3B). The puncture path was designed, and if the puncture path was blocked by a large sphenoid wing, the angle of the circular axis of the FR to the puncture direction was measured (Fig. 3B), and the individual radiofrequency needle bending device was used to bend the radiofrequency needle (Fig. $3 \mathrm{C}$ ) for percutaneous subzygomatic FR puncture. High $(50 \mathrm{~Hz})$ and low $(2 \mathrm{~Hz})$ frequency electrophysiological stimulation tests were performed after reaching the puncture target. If the numbness and muscle fibrillation of the $\mathrm{V} 2$ innervation region could be induced in $0.5 \mathrm{~mA}$, then, given appropriate propofol, radiofrequency thermocoagulation was applied (the parameters for thermocoagulation is $95^{\circ} \mathrm{C}$, last 120 s, repeat 2 or 3 times).

\section{Statistical Methods}

SPSS Version 17.0 software (IBM China Software Development Lab, SPSS Inc., Beijing, China) was used to analyze the data, and GraphPad Prism 6.0 (GraphPad Software Inc., La Jolla, CA) was used to map the analysis. The data were all expressed as mean \pm standard deviation $(X \pm s)$ or percentage (\%). The right and left sides of the same patient were paired using a sample $t$ test. The $t$ test was used for independent samples between 2 groups, whereas the probability of the puncture needle entering into the SOF, the ratio of entering into the FR, and ex ratio were tested by the chi-square test between groups. $P<0.05$ was considered significantly different. Institutional review board and ethics com- 
Table 2. Measurement in CT and $3 D$ reconstruction.

mittee approval was obtained from the institutional review board of the Jiaxing First Hospital (2017-188). The patients have consented to the submission of the study results.

\section{RESULTS}

\section{Imaging Data}

Two- and 3D anatomies of paranasal sinuses were analyzed in 88 cases. The shortest diameter of the FR (H) was $1.71 \pm 0.36 \mathrm{~mm}$; the minimum diameter was $1 \mathrm{~mm}$ and the maximum diameter was $2.9 \mathrm{~mm}$. The length of the FR tubes $(L)$ was $6.41 \pm 1.43 \mathrm{~mm}$; the maximum diameter was $10.9 \mathrm{~mm}$ and the minimum was $3.7 \mathrm{~mm}$. The distance between the external orifice of the FR and the SOF (FS) was $5.16 \pm 1.33 \mathrm{~mm}$; the minimum diameter was $2.8 \mathrm{~mm}$ and the maximum was $10.7 \mathrm{~mm}$. A paired sample t test on the left and right sides showed no significant difference in $\mathrm{H}, \mathrm{L}, \mathrm{FS}$, deviation angle of the misplacement into the SOF (SAF), and deviation angle of the misplacement into the OC (OAF) $(P>0.05)$; therefore, descriptive statistical analysis was performed.

The angle $A$ (between the radiofrequency needle and the sagittal plane) was $39 \pm 3.95^{\circ}$; the maximum was $51^{\circ}$ and the minimum was $31^{\circ}$. The ACT was 58.99 $\pm 6.23^{\circ}$; the maximum was $75^{\circ}$ and the minimum was $41^{\circ}$. The LS was $63.99 \pm 4.24 \mathrm{~mm}$; the maximum was 73 $\mathrm{mm}$ and the minimum was $52 \mathrm{~mm}$. The SAF was $2.96 \pm$ $0.71^{\circ}$; the minimum was $2^{\circ}$. The OAF was $4.95 \pm 0.73^{\circ}$, the minimum was $3^{\circ}$ (Table 2 ).

\section{Clinical Data Results of the 4 Groups}

The pre-NRS scores in the SB, SN, CB, and CN groups were $5.12 \pm 0.83,5.14 \pm 0.53,5.27 \pm 0.78$, and $5.29 \pm$ 0.58 , respectively, and the difference between scores was not statistically significant $(P>0.05)$. Moreover, the post-NRS scores in the CB group $(1.65 \pm 0.49)$ and the SB group $(2.56 \pm 1.00)$ were significantly lower compared with pre-NRS scores of the CB and the SB group, respectively $(P<0.001)$. In addition, the post-NRS scores in the $\mathrm{CB}$ group were significantly lower compared with postNRS of the SB group $(P<0.001)$ (Fig. 5A). The post-NRS scores in the SN group $(1.71 \pm 0.46)$ and the $\mathrm{CN}$ group $(1.68 \pm 0.59)$ were significantly lower compared with pre-NRS scores in the same groups $(P<0.001)$, whereas no significant difference in post-NRS scores were observed between the SN group and the CN group (Fig. $5 B)$. Furthermore, the total number of punctures $(4.47 \pm$

\begin{tabular}{|l|c|c|c|}
\hline Measurement & Mean & Maximum & Minimum \\
\hline H $(\mathrm{mm})$ & $1.71 \pm 0.36$ & 2.9 & 1 \\
\hline L $(\mathrm{mm})$ & $6.41 \pm 1.44$ & 10.9 & 3.7 \\
\hline FS $(\mathrm{mm})$ & $5.16 \pm 1.33$ & 10.7 & 2.8 \\
\hline LS $(\mathrm{mm})$ & $63.99 \pm 4.24$ & 73 & 52 \\
\hline A (degree) & $39 \pm 3.95$ & 51 & 31 \\
\hline ACT (degree) & $58.99 \pm 6.23$ & 75 & 41 \\
\hline SAF (degree) & $2.96 \pm 0.71$ & 4 & 2 \\
\hline OAF (degree) & $4.95 \pm 0.73$ & 6 & 3 \\
\hline
\end{tabular}

1.55) was significantly lower in the $\mathrm{SN}$ group compared with the CN group $(5.24 \pm 1.53)(P<0.05)$, whereas the total number of punctures in the SB group $(5.56 \pm 1.39)$ was not significantly different compared with the $C B$ group $(6.5 \pm 2.1)$ (Fig. $5 \mathrm{C}$ ). Additionally, the probability of misentry into the SOF in the SB group (40\%) was higher than that in the $C B$ group $(11.5 \%)\left(X^{2}=5.436\right.$; $P=0.02)$, whereas the probability of misentry into the SOF in the SN group (3.9\%) was lower compared with the CN group (14.7\%) $\left(X^{2}=3.139 ; P=0.076\right)$ (Fig. 5D). Also, the ratio of entering the FR in the SB group (40\%) was significantly lower compared with the $C B$ group $(100 \%)(P<0.05)$, whereas the ratio of entering the FR in the SN group (100\%) was not different from that in the CN group (100\%) (Fig. 5E).

\section{Discussion}

Proficiency in the anatomic structure related to the FR can help to avoid complications in CT-guided PIR ablation of the maxillary nerve through the FR employed to treat V2 TN. Three-dimensional (3D) reconstruction has definite guiding significance for clinical work $(18,19)$. Through the $3 D$ reconstruction and measurement of the FR and the SOF, the structural characteristics of the FR and the SOF can be better understood. According to previous studies, in percutaneous Hartel approach FO, balloon compression, and percutaneous radiofrequency thermocoagulation semilunar ganglion, the needle passes during the puncture through the inferior orbital fissure into the SOF and the external orifice of the $O C$, which can damage the optic nerve and lead to blindness $(20,21)$. It has been reported that with these approaches it is possible to enter into the $\mathrm{OC}$ at an angle of $16.5 \pm 2.9^{\circ}$ (22). In the present research, we found that the tip of the needle only needs to deviate from the normal trajectory $2.96 \pm 0.71^{\circ}$, and minimally $2^{\circ}$, to enter into the SOF and to cause damage of the 


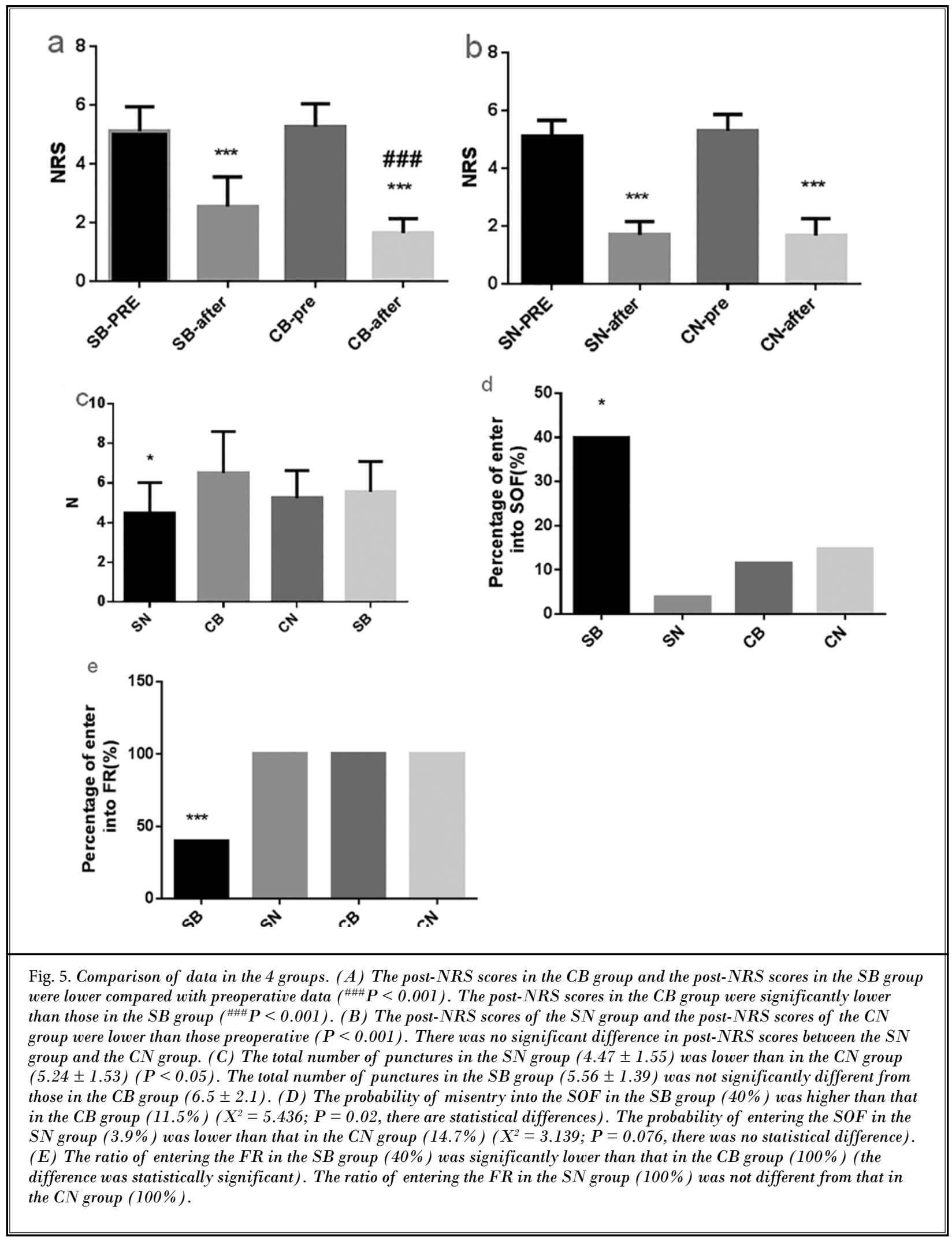


important vessels and nerves in the SOF. Also, when the upward deviation was $4.95 \pm 0.73^{\circ}$, and minimally $3^{\circ}$, the tip may enter into the $O C$ and cause optic nerve injury or even blindness $(23,24)$. The complications related to the optic nerve and nerve and blood vessels in the SOF more easily occur when the PIR ablation of the maxillary nerve is performed through the FR than with the percutaneous Hartel approach FO. Therefore, in clinical work, this issue should be properly addressed. Because the zygomatic nerve and other small branches may separate from the maxillary nerve in the inner FR (25), if the radiofrequency therapy is only applied at the external orifice of the FR, this can lead to partial neurologic branch missing and can cause radiofrequency thermocoagulation damage, thus leaving the treatment incomplete.

The analysis and comparison of clinical data revealed that for the patients with the blocking of the FR external orifice, the curved needle, which is personalized needle modification, can reduce the probability of entering into the SOF during the puncture, and increase the ratio of entering into the $F R$, thus achieving a definite therapeutic effect. For patients without blocking by the sphenoid wing, straight needles can reduce the number of needle adjustments, which in turn can reduce the total number of punctures, thereby reducing radiation exposure. In clinical work, we also found that even if the tip of the needle goes into the orbital fissure, with no radiofrequency thermal coagulation performed, it will not cause diplopia, strabismus, keratitis, mild vision loss, and other ocular complications. Nevertheless, if the SOF is mistaken for the FR during radiofrequency thermocoagulation treatment, it can certainly cause injury of the ophthalmic nerve, oculomotor nerve, trochlear nerve, and abducent nerve in the SOF, and even optic nerve injury in the OC (24) that can cause obvious damage to the patient's vision. Consequently, it is very important for the operator to distinguish the FR from the SOF. When it is established that the needle tip deviates from the intended puncture direction and points to the SOF through the external orifice of the $F R$, it is necessary to immediately stop the needle entry, to correct the puncture direction, or to use the curved needle technique to make the needle tip turn into the $\mathrm{FR}$, and thus prevent serious ocular complications after radiofrequency. The FR and its adjacent structures are very much complex, which is why it is necessary to fully understand the adjacent structure of the FR that can help to design the puncture path and reduce the risk of surgery and the incidence of related complications.
This study measured the angle of deviation from the FR to the $O C$ and angle of deviation from the FR to the SOF in CT-guided PIR ablation of the maxillary nerve through the FR to treat V2 TN. It has certain guiding significance for clinical workers. CT-guided PIR ablation of the maxillary nerve through the FR to treat V2 TN is an innovative technique for the treatment of TN (15). Our results suggested that for the patients with blocking of the external orifice in the puncture path, the personalized needle modification can increase the probability of entering the FR, obtaining better therapeutic effect, reducing the probability of entering into the SOF, and reducing the probability of orbital neurovascular injuries.

\section{Limitations}

This study has few limitations. The clinical data that included the number of cases whose external orifice of the FR was blocked by the large wing of the sphenoid bone was relatively small. Although the chi-square test statistical data were meaningful, the interval between cases was longer. The accidental error caused by the operator during clinical operation may not be eliminated. More clinical data should be collected to preserve the results in future work.

\section{Conclusions}

Because the $\mathrm{H}$ is $1.71 \pm 0.36 \mathrm{~mm}(1-2.9 \mathrm{~mm})$, the diameter of the radiofrequency needle should be < $1.0 \mathrm{~mm}$ so that the radiofrequency needle can enter into the FR. The length of the FR was $6.41 \pm 1.43 \mathrm{~mm}$ (3.7-10.9 mm), and the depth of the needle should be $<3.7 \mathrm{~mm}$ after reaching the external orifice of the FR to avoid the puncture tip into the middle cranial fossa. The distance from the SOF to the FR is $5.16 \pm 1.33 \mathrm{~mm}$ (2.8-10.7 $\mathrm{mm}$ ). When the tip of the needle points to the SOF, if the reentry depth is $<2.8 \mathrm{~mm}$ after reaching the external orifice of the FR, the SOF can be avoided.

In CT-guided PIR ablation of the maxillary nerve through the FR used to treat V2 TN, the angle between the CT scan plane and canthus ear line plane is 58.99 $\pm 6.23^{\circ}$, which can make the puncture point, puncture target, and puncture path at the same CT plane. The average depth of radiofrequency needle puncture is 63.99 $\pm 4.24 \mathrm{~mm}$, the maximum is $73 \mathrm{~mm}$, and the minimum is $52 \mathrm{~mm}$. The average of angle between needle and sagittal plane is $39 \pm 3.95^{\circ}$, maximum is $51^{\circ}$, minimum is $31^{\circ}$. The angle of deviation into the SOF is $2.96 \pm 0.71^{\circ}$ and the minimum is $2^{\circ}$, much smaller than $A$ and ACT. An inexperienced physician is very likely to enter the 
SOF, causing vascular and nerve damage in the SOF. When the tip of the needle deviates from $4.95 \pm 0.73^{\circ}$, the minimum is $3^{\circ}$, it may enter the $O C$ and cause optic nerve injury or even blindness.

In CT-guided PIR ablation of the maxillary nerve through the FR used to treat V2 TN in patients with blockage in the path of puncture FR, the treatment of radiofrequency thermocoagulation with personalized needle modification can improve the ratio of entering into the FR $(100 \% / 40 \%)$, the curative effect $(1.65 \pm$ $0.49 / 2.56 \pm 1.00)$, and can reduce the probability of the puncture needle entering into the SOF $(11.5 \% / 40 \%)$; for the patients without blockage in the path of puncture FR, the total number of punctures $(4.47 \pm 1.55 / 5.24$ \pm 1.39 ) and the radiation dose of the patients and medical staff can be reduced by using a straight needle.

\section{Acknowledgments}

The authors want to thank members of the Department of Anesthesiology and Pain Medical Center in Jiaxing city for the acquisition of 136 patient treatment dates. Author contributions: Yajing Chen, Qiang Zhu, and Bing Huang had the original idea for the manuscript and collected the data. Qianying Liu, Qiuli He, and Yingbing Yao analyzed the data. Yajing Chen reviewed the literature for the introduction and drafted the manuscript. Ming Yao, Chunyan Zhu, Kang An, and Wei Chen revised the manuscript. Bing Huang assisted in drafting the manuscript, revision of the text, and approved the final manuscript. All authors read and approved the final manuscript.

\section{References}

1. Amagasaki K, Abe S, Watanabe S, Naemura K, Nakaguchi $\mathrm{H}$. Trigeminal neuralgia caused by a trigeminocerebellar artery. J Neurosurg 2014; 121:940-943.

2. Ko AL, Lee A, Raslan AM, Ozpinar A, McCartney S, Burchiel KJ. Trigeminal neuralgia without neurovascular compression presents earlier than trigeminal neuralgia with neurovascular compression. J Neurosurg 2015; 123:1519-1527.

3. Ko AL, Ozpinar A, Lee A, Raslan AM, McCartney S, Burchiel KJ. Long-term efficacy and safety of internal neurolysis for trigeminal neuralgia without neurovascular compression. ] Neurosurg 2015; 122:1048-1057.

4. Headache Classification Committee of the International Headache Society (IHS). The international classification of headache disorders, 3 rd edition (beta version). Cephalalgia 2013; 33:629-808.

5. Love S, Coakham HB. Trigeminal neuralgia: Pathology and pathogenesis. Brain 2001; 124:2347-2360.

6. Vorenkamp KE. Interventional procedures for facial pain. Curr Pain Headache Rep 2013; 17:308.
7. Reddy VK, Parker SL, Patrawala SA, Lockney DT, Su PF, Mericle RA. Microvascular decompression for classic trigeminal neuralgia: Determination of minimum clinically important difference in pain improvement for patient reported outcomes. Neurosurgery 2013; 72:749-754.

8. Liao C, Visocchi M, Yang M, Liu P, Li S, Zhang W. Pulsed radiofrequency: A management option for recurrent trigeminal neuralgia following radiofrequency thermocoagulation. World Neurosurg 2017; 97:760.e5-76o.e7.

9. Udupi BP, Chouhan RS, Dash HH, Bithal PK, Prabhakar H. Comparative evaluation of percutaneous retrogasserian glycerol rhizolysis and radiofrequency thermocoagulation techniques in the management of trigeminal neuralgia. Neurosurgery 2012; 70:407-412; discussion 412-403.

10. Martin S, Teo M, Suttner N. The effectiveness of percutaneous balloon compression in the treatment of trigeminal neuralgia in patients with multiple sclerosis. J Neurosurg 2015; 123:1507-1511.

11. Bender M, Pradilla G, Batra S, See A,
Bhutiani N, James C, Carson BS, Lim M. Effectiveness of repeat glycerol rhizotomy in treating recurrent trigeminal neuralgia. Neurosurgery 2012; 70:11251133; discussion 1133-1134.

12. Helis CA, Lucas JT Jr, Bourland JD, Chan MD, Tatter SB, Laxton AW. Repeat radiosurgery for trigeminal neuralgia. Neurosurgery 2015; 77:755-761.

13. Martinez Moreno NE, Gutierrez-Sarraga J, Rey-Portoles G, Jimenez-Huete A, Martinez Alvarez R. Long-term outcomes in the treatment of classical trigeminal neuralgia by gamma knife radiosurgery: A retrospective study in patients with minimum 2-year follow-up. Neurosurgery 2016; 79:879-888.

14. Wan Q, Zhang D, Cao X, Zhang Y, Zhu M, Zuo W. CT-guided selective percutaneous radiofrequency thermocoagulation via the foramen rotundum for isolated maxillary nerve idiopathic trigeminal neuralgia. J Neurosurg 2018; 128:211-214.

15. Huang B, Yao M, Feng Z, Guo J, Zereshki A, Leong M, Qian X. CT-guided percutaneous infrazygomatic radiofre- 
quency neurolysis through foramen rotundum to treat $\mathrm{V}_{2}$ trigeminal neuralgia. Pain Med 2014; 15:1418-1428.

16. Kanpolat $Y$, Savas A, Bekar A, Berk C. Percutaneous controlled radiofrequency trigeminal rhizotomy for the treatment of idiopathic trigeminal neuralgia: 25year experience with 1,600 patients. Neurosurgery 2001; 48:524-532.

17. Chen Z, Ma J, Xie KY, Huang B, Yao M, Fei $Y$, Zhang L. A study of the relations of foramen rotundum structure direction and the approach of percutaneous puncturing of radiofrequency thermocoagulation for treating $V_{2}$ of primary trigeminal neuralgia. Zhonghua $Y i$ Xue Za Zhi 2018; 98:436-439.

18. Fang L, Ying S, Tao W, Lan M, Xiaotong $Y, N a n$ J. 3D CT-guided pulsed radiofrequency treatment for trigeminal neuralgia. Pain Pract 2014; 14:16-21.
19. Guo Z, Wu B, Du C, Cheng M, Tian Y. Stereotactic approach combined with ${ }_{3} \mathrm{D} C T$ reconstruction for difficult-toaccess foramen ovale on radiofrequency thermocoagulation of the Gasserian ganglion for trigeminal neuralgia. Pain Med 2016; 17:1704-1716.

20. Egan RA, Pless M, Shults WT. Monocular blindness as a complication of trigeminal radiofrequency rhizotomy. Am J Ophthalmol 2001; 131:237-240.

21. Agazzi $S$, Chang $S$, Drucker MD, Youssef AS, Van Loveren HR. Sudden blindness as a complication of percutaneous trigeminal procedures: Mechanism analysis and prevention. J Neurosurg 2009; 110:638-641.

22. Alvernia JE, Sindou MP, Dang ND, Maley $\mathrm{JH}$, Mertens P. Percutaneous approach to the foramen ovale: An anatomical study of the extracranial trajectory with the incorrect trajectories to be avoided. Acta Neurochir 2010; 152:1043-1053.

23. Mori K, Yamamoto T, Nakao Y, Esaki T. Development of artificial cranial base model with soft tissues for practical education. Oper Neurosurg 2010; 66:onsE339-onsE341.

24. Froelich SC, Aziz KM, Levine NB, Theodosopoulos PV, van Loveren HR, Keller JT. Refinement of the extradural anterior clinoidectomy: Surgical anatomy of the orbitotemporal periosteal fold. Neurosurgery 2007;61:179-185.

25. Elhadi AM, Zaidi HA, Yagmurlu K, Ahmed S, Rhoton AL Jr, Nakaji P, Preul MC, Little AS. Infraorbital nerve: A surgically relevant landmark for the pterygopalatine fossa, cavernous sinus, and anterolateral skull base in endoscopic transmaxillary approaches. J Neurosurg 2016; 125:1460-1468. 
